Article to be presented at the

17th International Conference on the Application of Accelerators

in Research and Industry CAARI 2002

\title{
Engineering Properties of Superhard Films with Ion Energy and Post-Deposition Processing
}

\author{
Othon R. Monteiro ${ }^{(1)}$ and Marie-Paule Delplancke-Ogletree ${ }^{(2)}$ \\ (1) Lawrence Berkeley National Laboratory, University of California, \\ Berkeley, California 94720 \\ (2) Université Libre de Bruxelles \\ Department of Industrial Chemistry \\ Brussels, Belgium
}

October 2002

Contact Author: $\quad$ Dr. Othon R. Monteiro

Lawrence Berkeley National Laboratory

Mail Stop 53-004

One Cyclotron Rd.

Berkeley, CA 94720 USA

e-mail: ORMonteiro@lbl.gov

FAX: (510) 486-4374

This work was supported by the U.S. Department of Energy, under contract No. DE-AC03-76SF00098. 


\title{
Engineering Properties of Superhard Films with Ion Energy and Post-Deposition Processing
}

\author{
Othon R. Monteiro $^{(1)}$ and Marie-Paule Delplancke-Ogletree \\ (1) Lawrence Berkeley National Laboratory, University of California, Berkeley, CA 94720, USA \\ (2) Université Libre de Bruxelles, Department of Industrial Chemistry, Brussels, Belgium
}

\begin{abstract}
Recent developments in plasma synthesis of hard materials using energetic ions are described. Metal Plasma Immersion Ion Implantation and Deposition (MePIIID) has been used to prepare several hard films: from diamondlike carbon (DLC) to carbides, from nitrides to oxides. The energy of the depositing species is controlled to maximize adhesion as well as to change the physical and chemical properties of the films. Adhesion is promoted by the creation of a graded interface between the film and the substrate. The energy of the depositing ions is also used to modify and control the intrinsic stresses and the microstructure of the films. The deposition is carried out at room temperature, which is important for temperature sensitive substrates. A correlation between intrinsic stresses and the energetics of the deposition is presented for the case of DLC films, and means to reduce stress levels are discussed.
\end{abstract}

\section{INTRODUCTION}

The microstructure and properties of thin films strongly depend on the deposition process and process conditions used[1,2]. In addition to the conventional process parameters such as pressure, composition of the gas phase and substrate temperature, there has been increasing interest in controlling the energy of the depositing species[3].

One of the most successful examples of using energetic particles (atoms or ions) in materials synthesis is the deposition of diamondlike carbon (DLC) films. The term DLC in this article will be used to describe non-hydrogenated amorphous carbon with a significantly high content of tetragonal bonding. Such a high content of $\mathrm{sp}^{3}$ bonding can only be achieved with processes that make use of precursors with hyperthermal energies. In addition to affecting the chemistry, such as in the case of DLC, energetic ions can also promote control of grain size, texture and stress in films.

Traditionally DLC has been prepared by Pulsed Laser Deposition (PLD) [4], Filtered Cathodic Arc (FCA) $[5,6]$ and Mass Selected Ion Beam (MSIB)[7]. DLC is currently used as overcoats in magnetic disks and heads, as an ultra thin layer (less than $10 \mathrm{~nm}$ ), for protection of the magnetic media against wear and environmental degradation. In several applications, thicker films are necessary, and in these cases, it is important to reckon with the level of intrinsic stresses.
Typically, it has been observed that there is a correlation between the level of stresses and the $\mathrm{sp}^{3}$ content in these films, which define their diamondlike properties[5,8,9]. Since the as-deposited DLC films with highest $\mathrm{sp}^{3}$ content have very high levels of intrinsic stresses (as high as $10 \mathrm{GPa}$ ), thick films have tendency to delaminate from the substrate.

In this article, we describe different means of producing DLC films with reduced stress levels. Those include the use of alloying elements, thermal annealing and ion bombardment.

\section{DEPOSITION PROCESS}

The films used in this investigation were prepared using Filtered Cathodic Arc deposition with a simultaneous application of a pulsed bias voltage to the substrate. The process, named Metal Plasma Immersion Ion Implantation and Deposition (MePIIID) has been described in detail elsewhere[10,11,12],. The plasma source was operated in a pulsed mode, with pulse duration between 5 to $50 \mathrm{~ms}$. During each arc pulse a train of negative bias voltage pulses was applied to the substrate to provide additional energy to depositing ions. Applied bias voltages varied from $0 \mathrm{~V}$ to $-2000 \mathrm{~V}$, and the duty cycle varied from 0 to $100 \%$. (0 meaning no bias and $100 \%$ meaning dc bias). Figure 1 shows a schematic representation of the deposition set-up: 
(a)

(b)

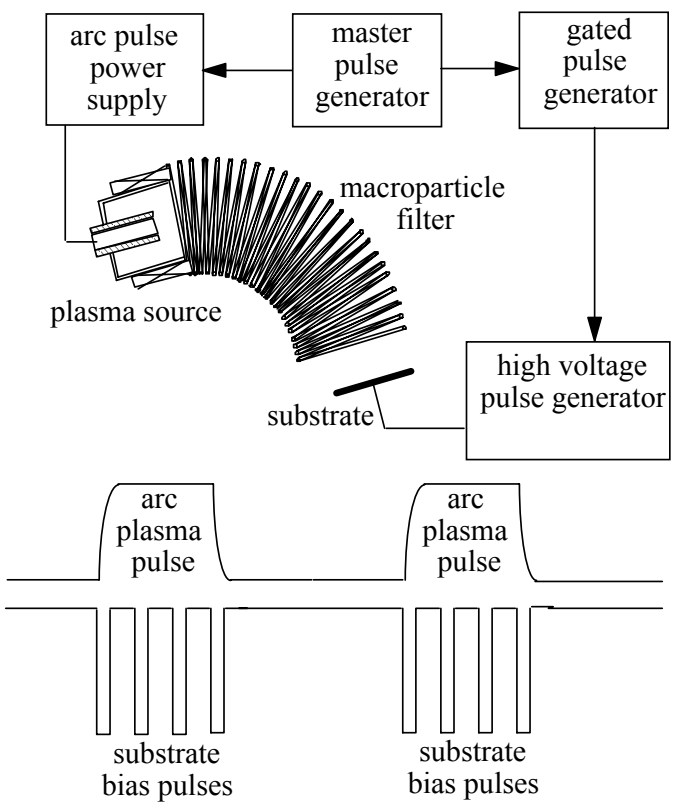

FIGURE 1: Schematic representation of Metal Plasma Ion Immersion Implantation and Deposition process: (a) source and electronics (b) arc current and bias voltages.

In the case of films containing more than one element (such as DLC:W), we used either two plasma sources. Although not discussed here, nitrides or oxides can be produced using one or several plasma sources and carrying out the deposition in a background gas pressure. In order to minimize collisions, the background gas pressure during deposition in the presence of reactive gases was maintained below $10^{-4}$ Torr.

Film properties were characterized by several different techniques: Transmission (TEM) and Scanning (SEM) Electron Microscopy, Rutherfordbackscattering Spectrometry (RBS), Auger Electron Spectroscopy (AES) and X-ray Photoelectron Spectroscopy. (XPS) Intrinsic stresses were calculated using Stoney's equation[13] after measuring the change in curvature radius of the coated substrates. Hardness and modulus were determined by nanoindentation, using a Hysitron Picoindenter.

\section{EFFECT OF ENERGETIC PARTICLES}

As mentioned in the Introduction, the properties of the deposited film are strongly affected by the energy of the depositing particles (ions or atoms)[3]. The plasma stream that is directed to the substrate during the deposition process mainly consists of a mixture of ions and electrons; neutrals are practically absent. The total energy of the depositing ions is a function of the chemical species of the cathode and the bias voltage applied to the substrate.

Energetic species impinging onto a substrate can dislodge adsorbed impurity atoms, and be incorporated below the substrate surface. Ion energies greater than $25 \mathrm{eV}$ are typically sufficient to promote desorption; sub-surface implantation (aka sub-plantation); and ion stitching. These processes promote adhesion between film and substrate.

The energy of the depositing species also has great effect on the surface mobility of adsorbed atoms. Surface mobility is a thermally activated process, which can be enhanced either by momentum transfer to adsorbed atoms, or by creation of surface defects.

Other important properties that depend on the energy of the incident particles are texturing and intrinsic stresses. Texturing is defined as the preferential orientation of individual grains of a material in one or several crystallographic directions. Texturing depends on surface mobility, sputtering yield and intrinsic stresses, and all of these depend on the energy of depositing species.

The dependence of stress on the incident energy is complex. Polycrystalline films prepared with low energy species tend to be under tensile stress resulting from grain coalescence, or low density grain boundaries. Energetic deposition leads usually to compressive stresses due to the incorporation of interstices. Judicious choice of energies can result in stress free films, or films with very small stress. Notice that although slightly compressive films are advantageous to improve resistance to fatigue and resistance to environmental attack, highly compressive films lead to premature failure by delamination.

\section{HARD COATINGS:}

The properties of DLC films prepared by energetic deposition strongly depend on ion energy $[5,9,10,14]$. Figure 2 shows the correlation between the film density and the carbon ion energy. In general, hardness, $\mathrm{sp}^{3}$ content and stress have similar dependences, i.e. they all achieve maximum values near $-100 \mathrm{eV}$. A recent publication has challenged the direct correlation between stress and $\mathrm{sp}^{3}$ content [15].

The challenge facing broader implementation of DLC is the high level of intrinsic stresses, which may reach values in excess of $10 \mathrm{GPa}$ in the hardest films. 
Several approaches have been proposed to reduce intrinsic stresses in DLC films, and are discussed in the following.

Once DLC was shown to be thermally stable in vacuum up to temperatures up to $700^{\circ} \mathrm{C}$, with very little change in bonding characteristics [16,17], it has been demonstrated that vacuum annealing could reduce intrinsic stresses to levels below $1 \mathrm{GPa}$ or better $[16,18]$. The mechanism that actually leads to stress relaxation is still under discussion. It is likely to be associated with conversion of bonds in a small number of $\mathrm{C}$ atoms in the film. Repetitive growth-annealing steps can in principle be used to produce thick films while keeping the level of stresses low. Albeit successful in laboratory scale, this approach is slow to produce film thickness of several tens of microns.

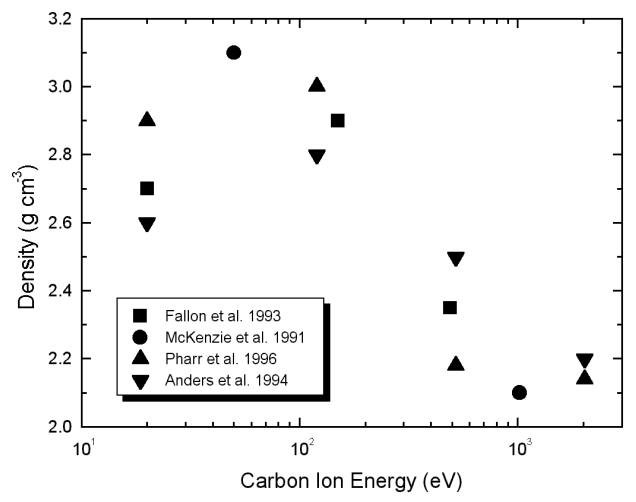

FIGURE 2: Density of non-hydrogenated DLC produced by filtered cathodic arc deposition as a function of the energy of the depositing $\mathrm{C}$ ion.

Another proposed process to mitigate the high level of intrinsic stresses in DLC has been the use of multilayers of DLC consisting of fully developed layers of hard and soft DLC [19]. In their work, hard DLC refers to the films with hardness and intrinsic stress around $60 \mathrm{GPa}$ and $10 \mathrm{GPa}$ respectively, and soft films to those with hardness and stresses around 25 $\mathrm{GPa}$ and $3 \mathrm{GPa}$ respectively. The properties of the resulting multilayer material are some average of the properties of the individual layers.

Yet another approach to produce superhard carbon films with low intrinsic stress has been investigated [20]. According to this approach, carbide forming elements such as refractory metals or silicon are added during the deposition of the DLC film. Incorporation of a few percent of $\mathrm{W}$ on the DLC matrix, for instance, led to large reduction in stress with somewhat small decrease in hardness, as shown in Figure 3 (a) and (b).
In some cases, e.g. at medium and high bias voltages, small addition of $\mathrm{W}$ resulted in an increase in hardness.

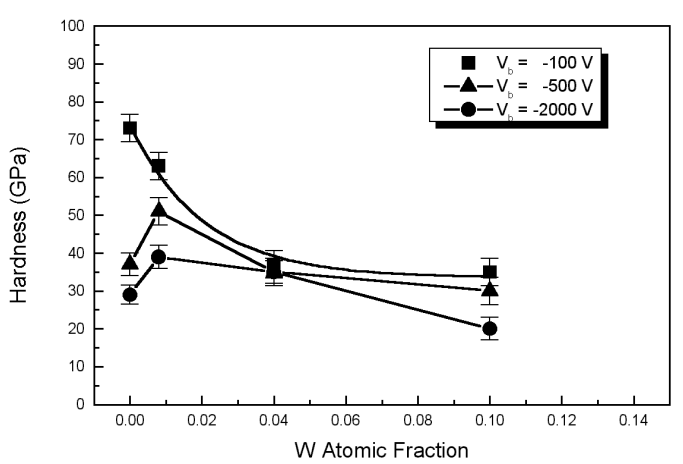

FIGURE 3(a): Hardness of DLC:W at several W content prepared using different bias voltages applied to the substrate

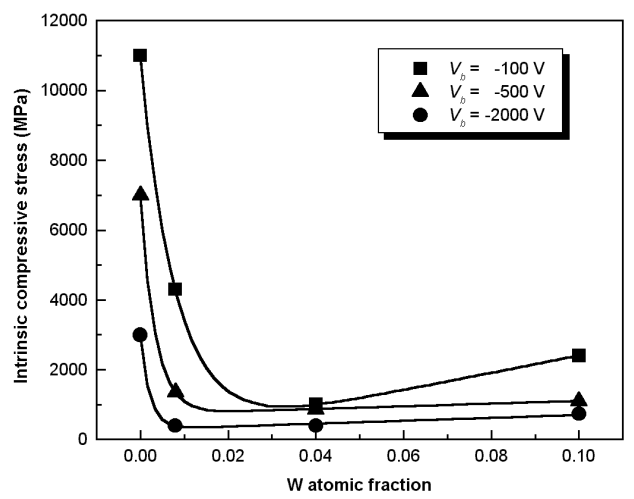

FIGURE 3(b): Intrinsic stress of DLC:W at several W content prepared using different bias voltages applied to the substrate

The decrease in intrinsic stress at very small addition of $\mathrm{W}$ is attributed mainly to the higher energy of the incident $\mathrm{W}$ ions, when compared to $\mathrm{C}$ ions. The mean charge state of $\mathrm{W}$ is larger than that of $\mathrm{C}$, and so are their native and ionization energies.

Thermal annealing has also been successfully used to decrease intrinsic stresses in the DLC:Me films. Figure 4 shows the evolution of stress in the case of Ti and $\mathrm{W}$ containing DLC upon annealing in Argon atmosphere up to $500^{\circ} \mathrm{C}$. When the starting stress level is high, such as in the cases where the bias voltage was $-100 \mathrm{~V}$, significant relaxation in intrinsic stresses occurs. On the other hand, the stress level remained 
practically unchanged when the films were prepared at $-500 \mathrm{~V}$ bias voltage.

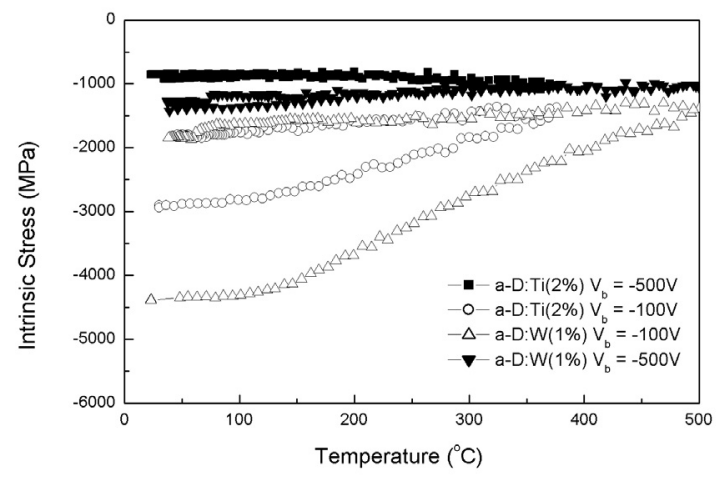

FIGURE 4: Evolution of stress in DLC:Ti and DLC:W films prepared with bias voltages of $-100 \mathrm{~V}$ and $-500 \mathrm{~V}$. .

The same rational that was used to use soft-hard DLC multilayers has inspired the use of multilayers consisting of alternating layers of DLC and DLC:Me. There are even some advantages about using the $\mathrm{Me}$ doped films instead of the pure $\mathrm{C}$ films.

As an example of such engineered structure, DLC - DLC:Ti multilayers were prepared and tested. The results of the hardness are presented in Figure 5. The total thickness of the multilayers were approximately (determined from RBS data):

\begin{tabular}{|c|c|}
\hline DLC $-3($ TiC-DLC) & $70 \mathrm{~nm}$ \\
\hline DLC $-2($ TiC-DLC $)$ & $150 \mathrm{~nm}$ \\
\hline DLC - TiC - DLC & $300 \mathrm{~nm}$ \\
\hline
\end{tabular}

The decrease of the measured hardness with increasing indentation depth is consistent with the increase of the contribution of the inner layers (and the substrate). However, the large decrease observed in the thinnest multilayer results cannot be only attributed to the substrate effect. In fact, chemical analysis demonstrated that the individual layers were so thin that there was enough mixing in all the layers and the only pure DLC layer was the top $7 \mathrm{~nm}$.

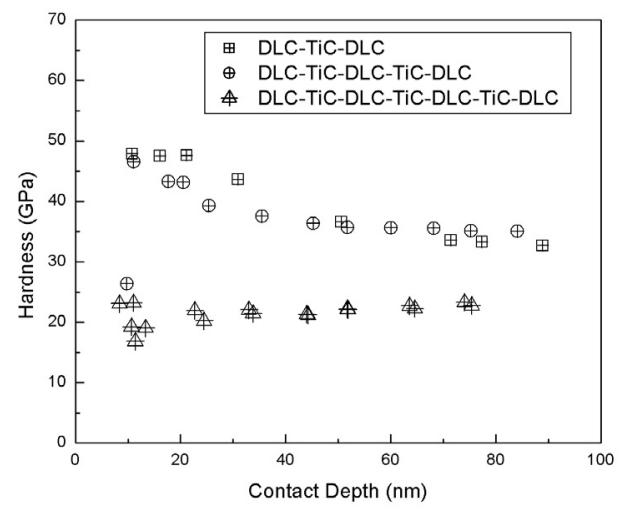

FIGURE 5: Hardness of three different multilayers consisting of alternating layers of DLC and DLC:Ti.

Recently it has been proposed that stress relaxation can be achieved by high-energy ion bombardment of the growing film[21,22]. The process is different from conventional Ion Beam Assisted Deposition (IBAD), in which ions that do not participate in the growth are used. Ion bombardment is accomplished here by accelerating the depositing ions through the plasma sheath resulting from the application of the high bias voltage. According to the proposed mechanism, local stress relaxation occurs primarily within the thermal spike volume.

\section{DISCUSSIONS:}

DLC has outstanding mechanical properties that make it a very interesting material to be used as protective coatings in applications where superior surface hardness is required. Its superior properties directly correlate with the $\mathrm{sp}^{3}$ content, and the only way to achieve high $\mathrm{sp}^{3}$ fraction is by carrying out the deposition using energetic ions. The major challenge for implementing thick DLC coatings in industrial applications is the management of the levels of intrinsic stresses.

Several techniques have been discussed. Stress can be decreased either in monolithic films by the incorporation of small amounts of alloying elements, by designing multilayer structures which alternatechemically distinct phases. Minimization of stress in multilayer structures is limited in the sense that the mean stress will be some average of the stresses of the individual layers. This solution is acceptable for a large range of applications, where intrinsic compressive stresses below 2 GPA are acceptable. 
In applications where compressive stresses below 1 $\mathrm{GPa}$ are required, the most promising methods include stresss relaxation by means of annealing achieved either by macroscopically raising the temperature for a predefined time interval, or by local stress relaxation via ion implantation. The latter appears to be more promising for large-scale industrial application.

\section{ACKNOWLEDGEMENTS}

This work was supported in part by the U.S. Department of Energy under contract number DEAC03-76SF00098.

\section{REFERENCES:}

1. Movchan BA, Demchishin AV. Fizika Metallov $i$ Metallovedenie 28, 653-659 (1969)

2. Thornton, J.A. J. Vac. Sci. Technol. 11, 666-670 (1974)

3. Monteiro, O. R. Ann. Rev. Mater. Sci. 31, 111-137 (2001)

4. Voevodin, A., Donley, M. S. and Zabinski, J. S. Surface and Coatings Technology. 52, 42-49 (1997)

5. Fallon P. J., Veerasamy, V. S., Davis, C. A., Robertson, J., Amaratunga, G. A. J. and Milne, W. I. Phys. Rev. B 48, 4777-4782 (1993)

6. Anders, S., Diaz, J., Ager, J. W., Lo, R. Y. and Bogy, D. B. Appl. Phys. Lett. 71, 3367 (1997)

7. Lifshitz, Y., Lempert, G. D. and Grossman, E. Phys. Rev. Lett. 72, 2753-2756 (1994)

8. Y. Lifshitz, Diamond and Related Materials 8, 16591676 (1999).

9 G. M. Pharr, D. L. Callahan, S. D. McAdams, T. Y. Tsui, S. Anders, A. Anders, J. W. Ager, III, I. G. Brown, C. S. Bhatia, S. R. P. Silva, and J. Robertson, Appl. Phys. Lett. 68, 779-81 (1996).

10. A. Anders, S. Anders, I. G. Brown, M. R. Dickinson, and R. A. MacGill, J. Vac. Sci. Technol. B 12, 815 820 (1994).

11. A. Anders, Surface Coatings Technol. 93, 157 - 167 (1997).

12. I. G. Brown, Ann. Rev. Mater. Sci. 28, 243 - 269 (1998).
13. G. G. Stoney, Proc. Roy. Soc. London A82, 172 - 176 (1909).

14. D. R. McKenzie, D. Muller, B. A. Palithorpe, Z. H. Wang, E. Kravtchinskaia, D. Segal, P. B. Lukins, P. J. Martin, G. Amaratunga, P. H. Gaskell, and A. Saeed, Diamond and Related Materials. 1, 51 - 59 (1991).

15. Ferrari, A. C., Rodil, S. E., Robertson, J. and Milne, W. I. Diamond and Related Materials 11, 994-999 (2002)

16. T. A. Friedmann, K. F. McCarty, J. C. Barbour, M. P. Siegal, and D. C. Dibble, Appl. Phys. Lett. 68, 1643 1645 (1996).

17. S. Anders, J. W. Ager, III, G. M. Pharr, T. Y. Tsui, and I. G. Brown, Thin Solid Films 308-309, 186-90 (1997).

18 Monteiro O. R., et al. J. Appl. Phys. 88, 2395-2399 (2000)

19. Anders S. Callahan DL. Pharr GM. Tsui TY. Bhatia CS Surface \& Coatings Technology. 94-95(1-3):189-194 (1997).

20 Monteiro O. R., Delplancke-Ogletree MP, Brown IGG. Thin Solid Films 342, 100-105 (1999)

21 Tarran, R. N., Montross, C. S. and McKenzie, D. R. Surface Coatings and Technology 136, 188-191 (2001)

22. Bilek, M. M. M., McKenzie, D. R., Tarrnat, R. N., Lim, S. H. M. and McCulloch, D. G. Surface Coatings and Technology 156, 136-142 (2002) 
